\title{
Optimization of Improvement Level for Second-Hand Product with Periodic Maintenance Schedule
}

\author{
Dae-Kyung $\operatorname{Kim}^{a} \cdot$ Jin Woo Kim ${ }^{b} \cdot$ Dong Ho Park ${ }^{c, 1}$ \\ ${ }^{a}$ Department of Statistics(Institute of Applied Statistics), Chonbuk National University \\ ${ }^{b}$ Department of Finance \& Information Statistics, Hallym University \\ ${ }^{c}$ Industry Academic Cooperation Foundation, Hallym University
}

(Received December 10, 2012; Revised January 11, 2013; Accepted January 23, 2013)

\begin{abstract}
Due to a growing demand for the second-hand product, especially for the expensive one, the warranty and maintenance policies for such products have been studied to improve the product reliability of late. In this paper we study a periodic maintenance model for the second-hand product which is purchased by the customer at the age of $x$. When purchased, the dealer provides a warranty of a fixed length during which the product is maintained periodically to reduce the failure rate of the product and thus, to improve the reliability after each maintenance is served. If a failure occurs between two successive maintenances, only minimal repair is conducted. As for the warranty policy, we adopt free non-renewing repair action on each failure, in addition to the periodic maintenance service during the warranty period. Thus, under the given warranty policy, all the maintenance and repair costs incurred during the warranty period are charged to the dealer. For the proposed periodic maintenance scheme, we formulate a cost model to evaluate the expected total cost charged to the dealer during the warranty period and derive an optimal upgrade level of the failure rate at each maintenance to minimize the expected total warranty cost from the perspective of the dealer. We also present numerical results for an optimal upgrade level based on the proposed methods.
\end{abstract}

Keywords: Second-hand product, minimal repair, periodic maintenance model, warranty policy, upgrade level, expected total cost.

\section{1. 서론}

현대사회의 극도로 경쟁적인 시장에서 중고 시스템 시장 또한 성장하고 경쟁적으로 변화하고 있다. 중 고 시스템 시장이 성장함에 따라 구입한 시스템의 고장 최소화를 선호하는 소비자들의 요구가 증가하 여 시스템 보증은 더욱 중요한 역할을 한다. 제조업체 또는 판매업체는 소비자들에게 시스템을 판매한 후 일정기간동안 발생하는 고장에 대하여 책임지는 보증 제도를 제공하는 것이 일반적이다. 그러나 판 매된 시스템에 대한 보증 제공은 시스템의 판매를 활성화 시키지만, 동시에 보증기간동안의 보전 및 수

This research is supported by Basic Science Research Program through the National Research Foundation of Korea (NRF) funded by the Ministry of Education, Science and Technology (2011-0022398).

${ }^{1}$ Corresponding author: Research Professor, Industry Academic Cooperation Foundation, Hallym University, Chuncheon, Gangwon-do 200-702, Korea. E-mail: dhpark@hallym.ac.kr 
리비용이 추가된다. 이러한 추가 비용은 시스템 점검 및 업그레이드, 예방보전정책 등 시스템의 신뢰성 을 향상시키는 작업을 통해 감소시킬 수 있다. 시스템 보증은 제조업체 또는 판매자가 소비자에게 지정 된 기간 동안 고장 시스템을 수리하거나 부분적 또는 전체적인 배상을 제공하는 계약이다. Blischke와 Murthy (2000)에 의하면 보증의 중요한 두 가지 역할은 결함이 있는 시스템에 대한 소비자와 소비자 의 과도한 요구에 대비하여 생산자 보호를 위한 보험, 생산자와 소비자에 의한 시스템 차별화를 위한 판촉활동이다. 20 세기 시작 이후 제조업체/판매업체에서 제공하는 보증기간은 꾸준하게 증가하고 있다 (Murthy와 Jack, 2003).

일반적으로 수리가 가능한 시스템에는 다양한 형태의 보증정책이 주어지기 때문에, 보증기간 동안에 제 공되는 새로운 시스템에 대한 사용자 측면의 교체정책 또는 예방보전정책과 관련된 연구가 최근까지 활 발하게 진행되고 있다. Nakagawa (1986)는 연속적이고 주기적인 보전 정책에 관한 최초의 연구결과 를 발표하였으며 그 이후 Zhang와 Jardine (1998)은 시스템의 최소 수리, 점검, 교체 3가지를 수행하 는 주기적 보전정책을 제안하였다. 다양한 형태의 보증정책에 관한 자세한 내용은 Blischke와 Murthy (1994, 1996)에서 찾을 수 있으며 대표적인 보증정책 문헌이다. Sahin과 Polatoglu (1996)는 보증기 간에 시스템의 고장이 발생되었을 경우에 시스템을 새 것으로 교체해주고 보증기간도 재생되는 재생보 증과 교체는 해주지만 보증기간은 재생되지 않는 비재생보증이 각각 제공되는 사용자 측면의 교체정책 에 대하여 살펴보았다. Jung과 Park (2003)은 Sahin과 Polatoglu (1996)가 제안한 보증기간이 종료 된 이후의 교체정책을 예방보전정책으로 확장하였으며 Chien (2008)은 재생무료교체보증이 주어진 시 스템에 대하여 일반적인 기령교체모형을 고려하였다, 재생무료교체보증은 보증기간동안 발생한 고장에 대하여 새로운 시스템으로 무료로 교체하고 교체된 시스템에 대하여 처음 판매시와 동일한 조건의 보증 이 적용되는 정책이며 기령교체는 일정한 나이에 도달한 시스템을 교체하는 정책을 의미한다. Jung 등 (2008)은 비용과 비 가동시간을 동시에 고려하여 비재생보증이 종료된 이후의 교체정책에 대하여 연구 하였다. 또한 Jung (2009)은 무료수리보증 뿐만 아니라 소비자에 의한 시스템의 사용기간에 비례하여 최소수리 비용의 일정 비율을 지불하는 비례수리보증인 경우의 최적 예방보전정책을 제안하였다.

본 논문에서는 중고 시스템에 대하여 제공된 보증기간 동안 고장 발생을 최소화시키기 위하여 판매자가 소비자로 하여금 주기적 예방보전을 수행하게 권장함으로써 보증서비스 비용을 최소화 할 수 있는 최적 보전정책과 보증비용에 대해 연구하려고 한다. 연구를 수행하기 위하여 본 논문에서는 주기적 예방보전 을 수행할 때 마다 고장률을 일정한 비율로 감소시키는 한편 고장률 패턴은 일정한 형태로 유지시키는 보전방법을 적용한다. 이러한 보전방법을 새로운 시스템에 적용시키는 연구는 과거에도 많은 연구자에 의하여 진행되어 왔으나 중고 시스템에 대하여는 매우 제한적인 연구만이 진행된 것으로 알려져 있다.

제 2 장에서는 이 논문에서 고려하는 주기적 예방보전정책을 정립하는데 필요한 가정과 보전방법을 설 명하고 중고 시스템의 판매 시에 제공되는 보증정책도 함께 기술한다. 또한 주기적 보전을 통하여 보전 되는 고장률함수를 유도함으로서 보증기간동안에 소요되는 전체 보증비용에 관한 공식을 유도한다. 제 3 장에서는 시스템의 수명이 와이블분포를 따른다는 가정 하에서 최적의 향상지수를 결정하는 방법을 유 도하며 제 4 장에서는 수치적 계산을 통하여 결과를 분석하고 관련모수들의 변화에 따르는 민감도분석을 제공한다. 이 연구를 통하여 얻어진 결론과 토의가 마지막 장에 주어진다.

\section{2. 중고 시스템의 주기적 보전정책}

이 장에서는 판매되는 중고시스템에 대하여 제공되는 보증정책과 보증기간동안에 수행되는 판매자 관점 에서의 주기적 예방보전정책에 관하여 논하고 보증비용을 계산하기 위한 비용모형을 정의하고 전체 보 증비용을 계산하기 위한 공식을 유도한다. 주기적 예방보전정책은 보증기간동안 일정한 주기로 수행되 


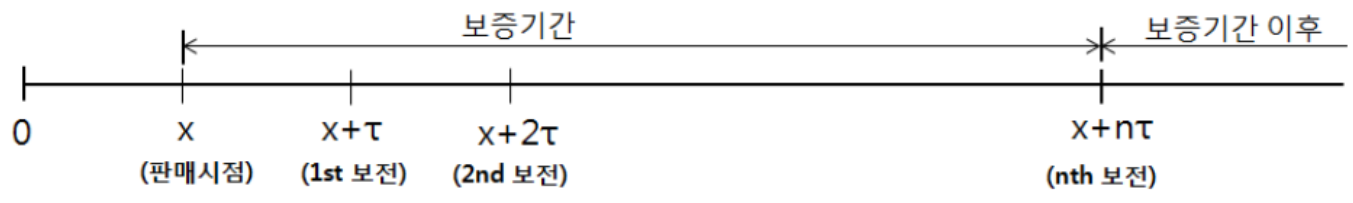

Figure 2.1. Maintenance model during warranty period

며 이 경우 매 예방보전시점에서 시스템의 고장률 패턴은 일정하게 유지하면서 고장률은 일정한 수준으 로 비례적으로 감소된다. 전체 보증비용은 판매직전에 수행되는 시스템의 업그레이드 비용, 예방보전시 의 보전비용 및 고장 발생시에 소요되는 최소수리 비용을 고려하여 계산된다.

\section{1. 주기적 보전정책 가정과 모형}

본 논문에서 고려하는 주기적 예방보전정책을 정립하기 위하여 다음과 같은 조건을 가정한다.

1) 시스템에는 일정한 길이의 보증기간 $\omega$ 가 주어진다.

2) 시스템 판매시의 나이는 $x$ 이고, 판매 이전의 고장에는 최소수리가 적용된다.

3) 보전은 시점 $k \tau, k=1,2, \ldots, n$ 에서 주기적으로 이루어지며, $\tau$ 는 보전의 주기이고 $n$ 은 보증기간동 안의 보전횟수이다. 따라서 보증기간의 길이 $\omega$ 는 $n \tau$ 와 같아지게 된다.

4) $n$ 번째 보전이 이루어지는 시점에서 보증은 만료된다.

5) 시스템은 각 보전 시점마다 향상지수 $0 \leq \alpha \leq 1$ 에 비례하여 고장률이 감소한다.

6) $h_{0}(t)$ 는 보전이 수행되기 이전의 초기 고장률함수이다.

7) 보전주기 사이에 시스템의 고장이 발생하면 최소수리를 수행하며 수리비용은 판매자가 부담한다.

8) 판매자는 중고 시스템의 보증기간 동안의 고장가능성을 감소시키기 위하여 판매 직전에 업그레이드 를 수행하며 소요된 비용을 부담한다. 업그레이드 비용은 $c_{0}$ 이다.

9) $n$ 번의 주기적 보전에 소요되는 보전비용은 판매자가 부담한다.

10) 보전과 최소수리, 교체를 위한 시간은 고려하지 않는다.

Figure 2.1은 이 논문에서 고려하는 중고 시스템의 보증기간 동안의 주기적인 보전모형을 설명해 준다. 중고 시스템이 새로운 구매자에 의해 구입된 시점에서의 시스템의 나이는 $x$ 라고 가정하고 보증기간 동 안 시점 $k \tau, k=1,2, \ldots, n$ 에서 주기적으로 보전이 이루어지며 $n$ 번째 보전시점에서 보증기간이 끝나 게 된다. 이 논문에서는 보증기간 $\omega$ 동안에 $n$ 번의 예방보전을 시행하는 것으로 가정하였으며 따라서 $\omega=n \tau$ 이고 시점 $x+n \tau$ 에서 시스템의 보증이 완료된다.

위에서 주어진 가정 아래에서 고려되는 보전정책은 Figure 2.2에 설명되어있다. 즉, 중고 시스템이 판 매되는 시점에서의 나이는 $x$ 이며 그 시점에서의 고장률함수는 $h_{0}(x)$ 로 가정한다. 그 이후 보증기간동 안 주기적인 예방보전을 수행하는데 매번의 예방보전 시점에서는 고장률함수의 모양은 그대로 유지되 지만 향상지수 $\alpha$ 의 크기에 비례하여 고장률함수가 감소되도록 보전이 수행되는데 이러한 보전방법이 Figure 2.2 의 그래프로 표시되어있다.

시스템의 나이가 $x+\tau$ 에 도달하면 즉, 첫 번째 예방보전시점에서 보전된 고장률, $h_{\alpha}(x+\tau)$ 은 다음과 같이 표시된다.

$$
h_{\alpha}(x+\tau)=\alpha h_{0}(x+\tau)+(1-\alpha) h_{0}(x)
$$




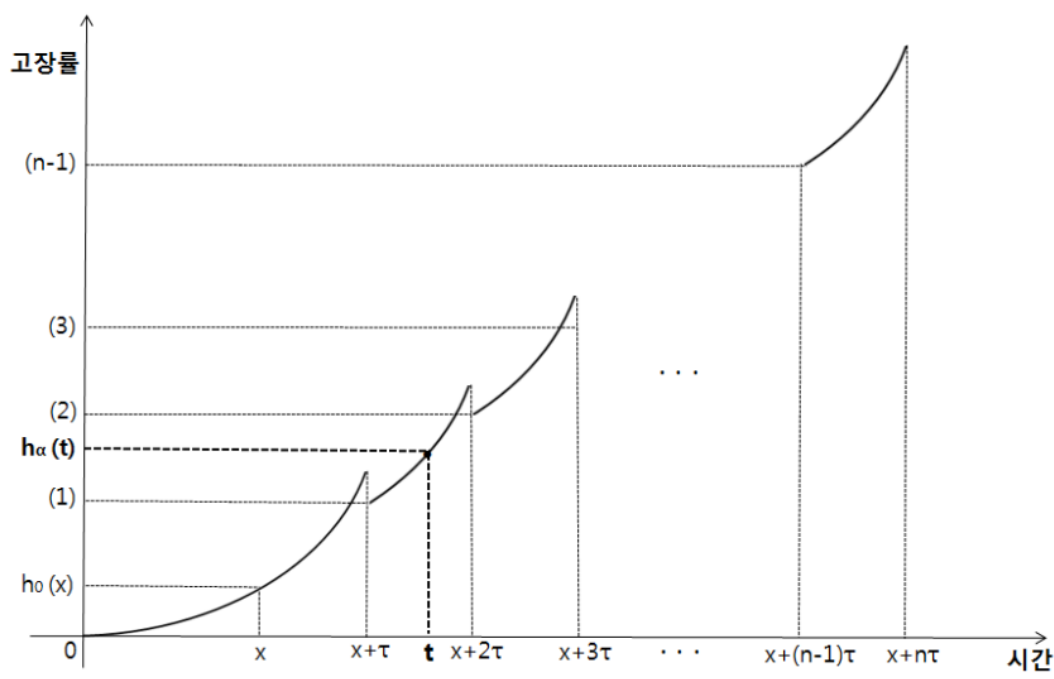

Figure 2.2. Graph of post-upgrade failure rate

여기에서 $h_{\alpha}(t)$ 는 시스템의 나이 $t$ 에서의 보전된 고장률을 나타낸다. 식 (2.1)로부터 $\alpha$ 의 값이 커질수 록 고장률의 감소크기는 작아지게 되며 따라서 $\alpha=0$ 이면 나이가 $x+\tau$ 에서의 보전된 고장률은 나이 $x$ 일 때의 초기 고장률로 최대로 감소되며, $\alpha=1$ 이면 $x+\tau$ 에서의 보전된 고장률이 $x+\tau$ 에서의 초기 고장률과 같게 되어 고장률 감소가 전혀 없게 된다. 따라서 주기적 예방보전 시에 감소되는 보전된 고장 률은 $\alpha$ 에 비례하게 되며 $\alpha$ 는 이 논문에서 향상지수라고 부르기로 한다.

나이가 $x+\tau$ 인 시점에서의 보전된 고장률함수는 $h_{\alpha}(x+\tau)$ 이며, 그 이후 나이 $x+2 \tau$ 시점까지의 고장 률 함수의 패턴은 나이 $x$ 에서 $x+\tau$ 구간에서의 고장률함수와 동일하며 따라서 $x+\tau \leq t<x+2 \tau$ 에 대 해서 보전된 고장률함수는 다음과 같이 표시된다.

$$
\begin{aligned}
h_{\alpha}(t) & =\alpha h_{\alpha}\left((x+\tau)^{-}\right)+(1-\alpha) h_{\alpha}(x)+\left[h_{0}(t-\tau)-h_{0}(x)\right] \\
& =\alpha\left[h_{0}(x+\tau)-h_{0}(x)\right]+h_{0}(x)+h_{0}(t-\tau)-h_{0}(x) \\
& =\alpha\left[h_{0}(x+\tau)-h_{0}(x)\right]+h_{0}(t-\tau),
\end{aligned}
$$

여기에서 $h_{\alpha}\left(t^{-}\right)=\lim _{\epsilon \rightarrow 0} h_{\alpha}(t-\epsilon)$ 을 의미한다. 시점 $(x+2 \tau)^{-}$에서의 고장률은 향상지수 $\alpha$ 를 적용 하면

$$
h_{\alpha}\left((x+2 \tau)^{-}\right)=\alpha\left[h_{0}(x+\tau)-h_{0}(x)\right]+h_{0}(x-\tau)
$$

이 얻어지며, 따라서 $x+2 \tau \leq t<x+3 \tau$ 에서의 보전된 고장률함수는

$$
\begin{aligned}
h_{\alpha}(t) & =\alpha h_{\alpha}\left((x+2 \tau)^{-}\right)+(1-\alpha) h_{\alpha}(x+\tau)+\left[h_{0}(t-2 \tau)-h_{0}(x)\right] \\
& =2 \alpha\left[h_{0}(x+\tau)-h_{0}(x)\right]+h_{0}(t-2 \tau)
\end{aligned}
$$

으로 얻어진다. 비슷한 방법으로 $x+k \tau \leq t<x+(k+1) \tau, k=1,2, \ldots, n$ 에서의 보전된 고장률함수 가 계산될 수 있으며 결과적으로 보전을 통한 고장률함수는 다음과 같이 얻어진다.

$$
h_{\alpha}(t)= \begin{cases}h_{0}(t), & x \leq t<x+\tau, \\ k \alpha\left\{h_{0}(x+\tau)-h_{0}(x)\right\}+h_{0}(t-k \tau), & x+k \tau \leq t<x+(1+k) \tau, k=1,2, \ldots, n .\end{cases}
$$


식 (2.2)를 이용하여 예방보전 구간별로 계산된 고장률함수는 다음과 같이 표시된다.

$$
h_{\alpha}(t)= \begin{cases}h_{0}(t), & x \leq t<x+\tau, \\ \alpha\left\{h_{0}(x+\tau)-h_{0}(x)\right\}+h_{0}(t-\tau), & x+\tau \leq t<x+2 \tau, \\ 2 \alpha\left\{h_{0}(x+\tau)-h_{0}(x)\right\}+h_{0}(t-2 \tau), & x+2 \tau \leq t<x+3 \tau, \\ 3 \alpha\left\{h_{0}(x+\tau)-h_{0}(x)\right\}+h_{0}(t-3 \tau), & x+3 \tau \leq t<x+4 \tau, \\ \quad \vdots & \vdots \\ (n-1) \alpha\left\{h_{0}(x+\tau)-h_{0}(x)\right\}+h_{0}(t-(n-1) \tau), & x+(n-1) \tau \leq t<x+n \tau .\end{cases}
$$

식 (2.3)은 보증기간 동안 판매자가 부담해야 되는 전체 보증비용을 유도하기 위한 중요한 함수로서 보 증기간동안에 발생하는 시스템의 고장횟수에 대한 기댓값을 계산하는데 사용된다.

\section{2. 보증기간동안의 보증비용 모형}

보증기간 동안 판매자에 의하여 소요되는 전체 보증비용은 판매직전에 수행되는 시스템의 업그레이드 비용, 주기적 예방보전시의 보전비용 및 고장 발생 시에 소요되는 최소수리 비용으로 구성된다. 여기에 서 업그레이드 비용은 비록 시스템의 판매직전에 소요되는 비용이지만 보증기간동안의 시스템의 고장을 감소하기 위한 보전활동으로 간주되어 보증비용에 포함되며 이 논문에서는 고정된 상수 $c_{0}$ 라고 가정한 다.

먼저 매번의 주기적 예방보전 시에 소요되는 보전비용은 판매시점에서의 중고 시스템의 나이 $(x)$ 와 향상 지수 $(\alpha)$ 에 종속되는 함수로서 다음과 같은 형태의 비용함수를 고려한다.

$$
c_{I}(\alpha, x)=\bar{c}(1-\alpha)^{\gamma} x^{\delta},
$$

여기서 $\bar{c}, \gamma, \delta>0$ 는 고정된 상수이고, $0 \leq \alpha \leq 1$ 는 향상지수이며 $\alpha=0$ 은 완전 업그레이드인 경우이 고, $\alpha=1$ 은 업그레이드가 전혀 되지 않은 보전을 의미한다. $\bar{c}$ 는 매번의 예방보전 시점에서 소요되는 평균 보전비용으로 고정된 상수이며 판매자에 의하여 지불된다.

보증기간동안의 최소수리 비용을 유도하기 위 하여는 중고 시스템의 보증기간동안 기대되는 평균고장 횟수를 먼저 계산하여야 한다. 보증기간동안 발생되는 중고 시스템의 고장횟수를 $N_{w}(x, \alpha, n)$ 으로 나타 내기로 하자. 이러한 고장횟수는 판매시의 시스템의 나이 $(x)$, 향상지수 $(\alpha)$ 와 예방보전횟수 $(n)$ 의 함수 이며 고장횟수에 대한 기댓값은 다음과 같이 계산될 수 있다.

$$
E\left[N_{w}(x, \alpha, n)\right]=\int_{x}^{x+w} h_{\alpha}(t) d t=\sum_{k=1}^{n} \int_{x+(k-1) \tau}^{x+k \tau} h_{\alpha}(t) d t,
$$

여기에서 $h_{\alpha}(t)$ 는 시스템의 나이 $t$ 에서의 보전된 고장률이며 보증기간동안의 보전된 고장률함수는 식 (2.3)에 표시되어 있다. 중고 시스템의 보증기간 동안 발생되는 고장 시에는 판매자의 부담에 의하여 최 소수리가 수행되는데 식 (2.5)를 이용하면 보증기간 동안의 최소수리비용, $C_{\omega}^{*}(x, \alpha, n)$ 에 대한 기댓값은 고장 횟수와 단위 최소수리 비용의 곱에 의해 다음과 같이 계산된다.

$$
E\left[C_{w}^{*}(x, \alpha, n)\right]=c_{m} \bullet E\left[N_{w}(x, \alpha, n)\right],
$$

여기에서 $c_{m}$ 은 최소수리에 소요되는 단위 수리비용을 나타내는 상수이다. 따라서 식 (2.4)와 식 (2.6)을 이용하면 보증기간 동안 판매자가 부담한 업그레이드 비용, 주기적 보전비용 및 고장발생 시 의 최소수리 비용을 합한 전체 보증비용에 대한 기댓값은 다음과 같이 얻을 수 있다.

$$
E\left[C_{w}(x, \alpha, n)\right]=c_{0}+n c_{I}(\alpha, x)+c_{m} E\left[N_{w}(x, \alpha, n)\right],
$$


여기서 $c_{0}$ 는 중고시스템의 판매 시에 판매자에 의하여 수행되는 초기 업그레이드에 소요되는 고정 비용, $c_{I}(\alpha, x)$ 는 식 $(2.4)$ 에 주어진 매 예방보전시의 단위 보전 비용, $c_{m}$ 은 단위 최소수리 비용을 나타낸다. 식 (2.7)은 보증기간동안에 지불되는 판매자의 총 보증비용에 대한 기댓값으로 이 비용을 최소화하는 결 정모수들을 찾아내는 것이 이 논문의 주요목표이며 다음 절에서 시스템의 수명이 와이블 분포를 따른다 는 가정 하에 최적의 주기적 예방보전을 위한 향상모수를 결정한다.

\section{3. 와이블 분포의 적용}

이 장에서는 시스템의 수명이 와이블 분포를 따른다고 가정하였을 때 식 (2.7)에서 유도된 보증기간동 안에 소요되는 전체 보증비용에 대한 기댓값을 계산한다. $T$ 가 시스템의 수명을 나타내는 확률변수이고 $T$ 는 다음과 같은 확률밀도함수를 가지는 와이블 분포를 따른다고 하자.

$$
f(t)=\lambda \beta(\lambda t)^{\beta-1} e^{-(\lambda t)^{\beta}}, \quad \lambda, \beta>0 .
$$

식 (3.1)을 이용하면 보전이 수행되기 이전의 $T$ 의 신뢰도 함수 $R_{0}(t)$ 와 고장률 함수 $h_{0}(t)$ 는 다음과 같 이 주어진다.

$$
\begin{aligned}
R_{0}(t) & =e^{-(\lambda t)^{\beta}}, \\
h_{0}(t) & =\lambda \beta \bullet(\lambda t)^{\beta-1} .
\end{aligned}
$$

따라서 주어진 $h_{0}(t)$ 를 이용하면 $k=1,2, \ldots, n$ 에 대하여 다음과 같은 관계식이 얻어진다.

$$
\begin{aligned}
h_{0}(x+\tau)-h_{0}(x) & =\lambda^{\beta} \beta\left[(x+\tau)^{\beta-1}-x^{\beta-1}\right], \\
h_{0}(t-k \tau) & =\lambda^{\beta} \beta(t-k \tau)^{\beta-1} .
\end{aligned}
$$

\section{1. 보증기간동안의 보증비용}

식 (3.2)를 식 (2.3)과 식 (2.5)에 대입하면 주어진 보증기간 동안의 평균 고장횟수에 대한 기댓값은 다 음과 같이 계산된다.

$$
\begin{aligned}
E\left[N_{w}(x, \alpha, n)\right]= & \int_{x}^{x+w} h_{\alpha}(t) d t \\
= & \int_{x}^{x+\tau} h_{\alpha}(t) d t+\int_{x+\tau}^{x+2 \tau} h_{\alpha}(t) d t+\cdots+\int_{x+(n-1) \tau}^{x+n \tau} h_{\alpha}(t) d t \\
= & \lambda^{\beta}\left[(x+\tau)^{\beta}-x^{\beta}\right] \\
& +\alpha \tau \lambda^{\beta} \beta\left[(x+\tau)^{\beta-1}-x^{\beta-1}\right]+\lambda^{\beta}\left[(x+\tau)^{\beta}-x^{\beta}\right] \\
& +2 \alpha \tau \lambda^{\beta} \beta\left[(x+\tau)^{\beta-1}-x^{\beta-1}\right]+\lambda^{\beta}\left[(x+\tau)^{\beta}-x^{\beta}\right] \\
& \vdots \\
& +(n-1) \alpha \tau \lambda^{\beta} \beta\left[(x+\tau)^{\beta-1}-x^{\beta-1}\right]+\lambda^{\beta}\left[(x+\tau)^{\beta}-x^{\beta}\right] \\
= & \sum_{k=0}^{n-1}\left\{k \alpha \tau \lambda^{\beta} \beta\left[(x+\tau)^{\beta-1}-x^{\beta-1}\right]+\lambda^{\beta}\left[(x+\tau)^{\beta}-x^{\beta}\right]\right\} .
\end{aligned}
$$


결과적으로 식 (2.4)와 식 (3.3)을 식 (2.7)에 대입함으로서 판매자가 보증기간 동안 지불해야 하는 전체 보증비용에 대한 기댓값은 다음과 같이 나타낼 수 있다.

$$
\begin{aligned}
E\left[C_{w}(x, \alpha, n)\right]= & c_{0}+n \bar{c}(1-\alpha)^{\gamma} x^{\delta}+c_{m} \lambda^{\beta} \sum_{k=0}^{n-1}\left\{k \alpha \beta \tau\left[(x+\tau)^{\beta-1}-x^{\beta-1}\right]+(x+\tau)^{\beta}-x^{\beta}\right\} \\
= & c_{0}+n \bar{c}(1-\alpha)^{\gamma} x^{\delta} \\
& +c_{m} n \lambda^{\beta}\left[(x+\tau)^{\beta}-x^{\beta}\right]+\alpha\left\{c_{m} \lambda^{\beta} \beta \tau \frac{n(n-1)}{2}\left[(x+\tau)^{\beta-1}-x^{\beta-1}\right]\right\} .
\end{aligned}
$$

\section{2. 향상지수 $\alpha$ 의 결정}

이 절에서는 3.1절에서 유도한 식 (3.4)의 $E\left[C_{w}(x, \alpha, n)\right]$ 을 최소화하는 $\alpha$ 의 값을 결정함으로서 최적의 주기적 예방보전정책을 제안한다. 여기에서 $E\left[C_{w}(x, \alpha, n)\right]$ 는 $x$ 와 $n$ 이 고정된 경우 $\alpha$ 의 함수로 간주되 며 따라서 1 차 및 2 차 도함수의 성질을 이용하여 이러한 함수를 최소화하는 $\alpha$ 의 값을 결정한다.

식 (3.4)로부터 얻어지는 $\alpha$ 에 관한 1 차 및 2 차 도함수는 다음과 같다.

$$
\begin{aligned}
\frac{d}{d \alpha} E\left[C_{w}(x, \alpha, n)\right] & =-n \bar{c} \gamma(1-\alpha)^{\gamma-1} x^{\delta}+c_{m} \lambda^{\beta} \beta \tau \frac{n(n-1)}{2}\left[(x+\tau)^{\beta-1}-x^{\beta-1}\right], \\
\frac{d^{2}}{d \alpha^{2}} E\left[C_{w}(x, \alpha, n)\right] & =n \bar{c} \gamma(\gamma-1)^{\gamma-2} x^{\delta} .
\end{aligned}
$$

식 (3.5)를 이용하여 $E\left[C_{w}(x, \alpha, n)\right]$ 을 최소화하는 $\alpha$ 의 값을 결정하기 위하여 다음과 같은 $\gamma$ 값의 범위 에 의한 3 가지 경우를 고려하며 여기에서 얻어지는 최적의 $\alpha$ 값을 $\alpha^{*}$ 로 표시한다.

a) $\gamma>1$ 인 경우

이 경우에는 모든 $0 \leq \alpha \leq 1$ 에 대해 $\left(d^{2} / d \alpha^{2}\right) E\left[C_{w}(x, \alpha, n)\right] \geq 0$ 이 성립되며 따라서 $E\left[C_{w}(x, \alpha, n)\right]$ 는 위로 오목인 함수(convex function)가 되고 $(d / d \alpha) E\left[C_{w}(x, \alpha, n)\right]=0$ 을 만족하는 $0<\alpha<1$ 의 값에서 최소값을 가지게 된다. 이 관계식으로부터 얻어지는 $\alpha^{*}$ 는 다음과 같이 표시된다.

$$
\alpha *=1-\left\{\frac{c_{m} \tau \lambda^{\beta} \beta(n-1)\left((x+\tau)^{\beta-1}-x^{\beta-1}\right)}{2 \bar{c} \gamma x^{\delta}}\right\}^{\frac{1}{\gamma-1}} .
$$

b) $\gamma=1$ 인 경우

$\gamma=1$ 인 경우에 $E\left[C_{w}(x, \alpha, n)\right]$ 는 다음과 같이 계산된다.

$$
\begin{aligned}
E\left[C_{w}(x, \alpha, n)\right]= & c_{0}+n \bar{c} x^{\delta} \\
& +c_{m} n \lambda^{\beta}\left[(x+\tau)^{\beta}-x^{\beta}\right]+\alpha\left\{c_{m} \lambda^{\beta} \beta \tau \frac{n(n-1)}{2}\left[(x+\tau)^{\beta-1}-x^{\beta-1}\right]-n \bar{c} x^{\delta}\right\} .
\end{aligned}
$$

식 (3.7)의 $E\left[C_{w}(x, \alpha, n)\right]$ 는 $\alpha$ 의 선형함수로서 $\alpha$ 의 계수가 0보다 크면(작으면) 즉, $c_{m} \lambda^{\beta} \beta \tau\{n(n-$ 1) $/ 2\}\left[(x+\tau)^{\beta-1}-x^{\beta-1}\right]>(<) n \bar{c} x^{\delta}$ 이면 $E\left[C_{w}(x, \alpha, n)\right]$ 는 $\alpha$ 의 증가(감소) 선형함수이며 결과적으로 $\alpha=0(\alpha=1)$ 에서 최소값을 가진다. 따라서 만일

$$
\begin{aligned}
& x^{\delta}<\frac{n-1}{2 \bar{c}} c_{m} \lambda^{\beta} \beta \tau\left[(x+\tau)^{\beta-1}-x^{\beta-1}\right] \text { 이면 } \alpha^{*}=0, \\
& x^{\delta}>\frac{n-1}{2 \bar{c}} c_{m} \lambda^{\beta} \beta \tau\left[(x+\tau)^{\beta-1}-x^{\beta-1}\right] \text { 이면 } \alpha^{*}=1
\end{aligned}
$$


의 해를 얻는다. 만일 $x^{\delta}=\{(n-1) /(2 \bar{c})\} c_{m} \lambda^{\beta} \beta \tau\left[(x+\tau)^{\beta-1}-x^{\beta-1}\right]$ 이면 $E\left[C_{w}(x, \alpha, n)\right]$ 는 $\delta$ 의 값에 관계없이 항상 일정한 값을 가지게 된다.

c) $\gamma<1$ 인 경우

이 경우에는 $\left(d^{2} / d \alpha^{2}\right) E\left[C_{w}(x, \alpha, n)\right]<0$ 이 되며 따라서 $E\left[C_{w}(x, \alpha, n)\right]$ 는 아래로 오목(concave function)인 $\alpha$ 의 함수이다. 결과적으로 $E\left[C_{w}(x, \alpha, n)\right]$ 는 $\alpha=0$ 또는 $\alpha=1$ 에서 최소값을 가지게 되며, $\alpha=0$ 과 $\alpha=1$ 에서의 $E\left[C_{w}(x, \alpha, n)\right]$ 을 비교함으로서 $\alpha^{*}$ 의 값이 0 과 1 중에서 결정된다.

\section{4. 수치적 계산과 결과분석}

3 장에서는 시스템의 수명이 와이블분포를 따른다는 가정 하에서 전체 보증비용에 대한 기댓값과 최적의 $\alpha$ 값을 결정하는 공식을 유도하였다. 이 장에서는 3 장에서 유도된 $\alpha^{*}$ 와 $E\left[C_{w}(x, \alpha, n)\right]$ 의 공식을 이용 한 수치적 계산결과를 제공하고 관련모수들의 변화에 따르는 민감도분석을 논의한다. 수치적 계산을 하 기 위하여 먼저 판매된 중고 시스템의 보증기간은 $\omega=2$ (단위: 년)로 가정하고 보증기간 동안 $n=4$ 의 주기적 예방보전을 수행한다고 하자. 그러면 보전주기는 6 개월이며 따라서 $\tau=0.5$ 가 된다. 보증기간 에 소요되는 비용은 임의의 값을 취하여 $c_{0}=500, \bar{c}=100, c_{m}=150$ 을 가정한다. 여기에서 $c_{0}$ 은 판 매직전에 소요되는 업그레이드 비용으로 평균보전비용 $(\bar{c})$ 이나 최소수리비용 $\left(c_{m}\right)$ 에 비하여 비교적 크다 고 가정 하는 것이 타당하다. 시스템의 수명분포인 와이블분포의 모수인 $\lambda$ 와 $\beta$ 는 각각 0.5 와 2 로 가정 한다. $\beta=2$ 는 고장률이 증가되는 수명분포를 나타내며 시스템의 평균수명을 2 년 보다 크게 하기 위하 여 $\lambda=0.5$ 로 가정하였다. 그 이외의 주요 모수들인 $x, \delta, \tau$ 등에 관하여는 $\alpha^{*}$ 와 $E\left[C_{w}(x, \alpha, n)\right]$ 에 미치 는 영향을 조사하기 위하여 민감도분석 결과를 제공한다.

1) $\gamma>1$ 인 경우에는 $E\left[C_{w}(x, \alpha, n)\right]$ 은 고정된 $x$ 와 $n$ 에 대하여 $\alpha$ 의 위로 오목인 함수이며 식 (3.4)와 식 (3.6)에 의하여 $\alpha^{*}$ 와 $E\left[C_{w}\left(x, \alpha^{*}, n\right)\right]$ 의 값이 결정된다. Table 4.1과 Table 4.2 는 $x$ 와 $\delta$ 값의 변화 에 따르는 $\alpha^{*}$ 와 $E\left[C_{w}\left(x, \alpha^{*}, n\right)\right]$ 의 값을 각각 나타낸다.

Table 4.1 과 Table 4.2 에 의하면 $\delta$ 가 증가하면 $\gamma$ 에 관계없이 $\alpha^{*}$ 의 값은 0 에 점점 가까워지고 $E\left[C_{w}\left(x, \alpha^{*}, n\right)\right]$ 은 감소한다. 또한 $x$ 값이 증가하면, 즉 판매시의 시스템의 나이가 많아지면 모든 경우에 $\alpha^{*}$ 와 $E\left[C_{w}\left(x, \alpha^{*}, n\right)\right]$ 는 증가하게 되며 특히 $x=1$ 인 경우에는 $\delta$ 의 값과 관계없이 $\alpha^{*}$ 와 $E\left[C_{w}\left(x, \alpha^{*}, n\right)\right]$ 의 값은 동일하다.

2) $\gamma=1$ 인 경우에는 식 (3.8)에 의하여 $\alpha^{*}=0$ 또는 $\alpha^{*}=1$ 의 값을 가진다. Table 4.3 에 의하면 $x<1$ 에 대해서는 $\delta$ 가 증가함에 따라 $\alpha=0$ 일 때의 비용은 감소되고 $\alpha=1$ 일 때의 비용은 모두 일 정하다. $\delta$ 의 값이 작을 때에는 $\alpha^{*}=1$ 이고 $\delta$ 의 값이 커지면 $\alpha^{*}=0$ 을 취한다. $x=1$ 이면 $\delta$ 와 관계 없이 $\alpha=0$ 일 때와 $\alpha=1$ 일 때의 비용이 항상 일정하며 $x>1$ 이면 전체 보증비용은 $\delta$ 에 관계없이 항상 $\alpha^{*}=1$ 의 값을 취한다.

3) $\gamma<1$ 인 경우에는 $E\left[C_{w}(x, \alpha, n)\right]$ 은 고정된 $x$ 와 $n$ 에 대하여 $\alpha$ 의 아래로 오목인 함수이며 따라서 $\alpha^{*}$ 의 값은 0 또는 1 이 된다. Table 4.4 는 이 경우의 $\alpha=0$ 과 $\alpha=1$ 에서의 전체 보증비용을 나타 내고 있으며 특히 $\gamma<1$ 이고 $\alpha=0$ 과 $\alpha=1$ 인 경우에는 $E\left[C_{w}(x, \alpha, n)\right]$ 은 $\gamma$ 의 값에는 무관하다. Table 4.4 는 $x<1$ 일 때의 비용은 $\delta$ 가 증가할수록 감소하고 $x=1$ 일 때의 비용은 $\delta$ 와 관계없이 항 상 일정하다는 것을 보이며 또한 $x>1$ 일 때의 비용은 $\delta$ 가 커질수록 증가한다. 따라서 $x<1$ 인 경우 에는 $\delta$ 의 값이 작을 때에는 $\alpha^{*}=1$ 이고 $\delta$ 의 값이 커지면 $\alpha^{*}=0$ 을 취하고 $x>1$ 인 경우에는 항상 $\alpha=0$ 에서 전체 보증비용이 최소가 된다. 
Table 4.1. Value of $\alpha^{*}$ for different combinations of $\delta$ and $x$ for $\gamma>1$

\begin{tabular}{|c|c|c|c|c|c|}
\hline \multirow{2}{*}{$\gamma$} & \multirow{2}{*}{$\delta$} & \multicolumn{4}{|c|}{$x$} \\
\hline & & 0.5 & 1.0 & 1.5 & 2.0 \\
\hline \multirow{4}{*}{1.5} & 0.3 & 0.947 & 0.965 & 0.972 & 0.977 \\
\hline & 0.6 & 0.920 & 0.965 & 0.978 & 0.985 \\
\hline & 1.0 & 0.860 & 0.965 & 0.984 & 0.991 \\
\hline & 2.0 & 0.437 & 0.965 & 0.993 & 0.998 \\
\hline \multirow{4}{*}{2.0} & 0.3 & 0.827 & 0.860 & 0.875 & 0.886 \\
\hline & 0.6 & 0.787 & 0.860 & 0.890 & 0.907 \\
\hline & 1.0 & 0.719 & 0.860 & 0.906 & 0.930 \\
\hline & 2.0 & 0.438 & 0.860 & 0.938 & 0.965 \\
\hline \multirow{4}{*}{3.0} & 0.3 & 0.660 & 0.694 & 0.712 & 0.724 \\
\hline & 0.6 & 0.623 & 0.694 & 0.729 & 0.751 \\
\hline & 1.0 & 0.567 & 0.694 & 0.750 & 0.783 \\
\hline & 2.0 & 0.388 & 0.694 & 0.800 & 0.847 \\
\hline \multirow{4}{*}{4.0} & 0.3 & 0.558 & 0.587 & 0.604 & 0.615 \\
\hline & 0.6 & 0.526 & 0.587 & 0.620 & 0.641 \\
\hline & 1.0 & 0.480 & 0.587 & 0.639 & 0.672 \\
\hline & 2.0 & 0.345 & 0.587 & 0.685 & 0.740 \\
\hline \multirow{4}{*}{5.0} & 0.3 & 0.487 & 0.513 & 0.528 & 0.538 \\
\hline & 0.6 & 0.460 & 0.513 & 0.542 & 0.561 \\
\hline & 1.0 & 0.421 & 0.513 & 0.560 & 0.590 \\
\hline & 2.0 & 0.311 & 0.513 & 0.602 & 0.656 \\
\hline
\end{tabular}

Table 4.2. Value of $E\left[C_{w}\left(x, \alpha^{*}, n\right)\right]$ for different combinations of $\delta$ and $x$ for $\gamma>1$

\begin{tabular}{|c|c|c|c|c|c|}
\hline \multirow{2}{*}{$\gamma$} & \multirow{2}{*}{$\delta$} & \multicolumn{4}{|c|}{$x$} \\
\hline & & 0.5 & 1.0 & 1.5 & 2.0 \\
\hline \multirow{3}{*}{1.5} & 0.6 & 721.97 & 798.68 & 874.19 & 949.43 \\
\hline & 1.0 & 719.73 & 798.68 & 874.41 & 949.67 \\
\hline & 2.0 & 703.91 & 798.68 & 874.74 & 949.92 \\
\hline \multirow{3}{*}{2.0} & 0.6 & 713.01 & 792.09 & 868.80 & 944.78 \\
\hline & 1.0 & 709.18 & 792.09 & 869.73 & 946.05 \\
\hline & 2.0 & 693.36 & 792.09 & 871.49 & 948.02 \\
\hline \multirow{3}{*}{3.0} & 0.6 & 696.73 & 777.04 & 854.67 & 931.35 \\
\hline & 1.0 & 692.52 & 777.04 & 856.25 & 933.76 \\
\hline & 2.0 & 679.07 & 777.04 & 859.70 & 938.52 \\
\hline \multirow{3}{*}{4.0} & 0.6 & 685.00 & 765.18 & 842.89 & 919.68 \\
\hline & 1.0 & 681.12 & 765.18 & 844.58 & 922.36 \\
\hline & 2.0 & 669.72 & 769.18 & 848.42 & 928.06 \\
\hline
\end{tabular}

\section{5. 결론}

이번 연구에서는 최근에 와서 많은 관심을 가지고 있는 중고 시스템의 보증정책과 보증기간동안의 최적 주기적 예방보전정책에 관하여 조사하였으며 특히 보증기간동안의 보증비용을 계산하기 위한 새로운 수 학적 모형을 정립하였다. 이 논문에서 고려한 보전정책은 매번의 주기적 보전 시에 향상지수에 비례하 여 시스템의 고장률을 감소시킴으로서 보증기간동안의 고장발생횟수를 줄이고 결과적으로 보증비용을 
Table 4.3. Value of $E\left[C_{w}(x, \alpha, n)\right]$ for different combinations of $\delta$ and $x$ for $\alpha=0, \alpha=1$ when $\gamma=1$

\begin{tabular}{|c|c|c|c|c|}
\hline$\gamma$ & $\delta$ & $x$ & $\alpha=0$ & $\alpha=1$ \\
\hline \multirow{20}{*}{1} & \multirow{4}{*}{0.5} & 0.5 & 895.34 & 725 \\
\hline & & 1.0 & 1087.50 & 800 \\
\hline & & 1.2 & 1155.68 & 830 \\
\hline & & 1.5 & 1252.40 & 875 \\
\hline & \multirow{4}{*}{1.0} & 0.5 & 812.50 & 725 \\
\hline & & 1.0 & 1087.50 & 800 \\
\hline & & 1.2 & 1197.50 & 830 \\
\hline & & 1.5 & 1362.50 & 875 \\
\hline & \multirow{4}{*}{1.5} & 0.5 & 753.92 & 725 \\
\hline & & 1.0 & 1087.50 & 800 \\
\hline & & 1.2 & 1243.31 & 830 \\
\hline & & 1.5 & 1497.35 & 875 \\
\hline & \multirow{4}{*}{2.0} & 0.5 & 712.50 & 725 \\
\hline & & 1.0 & 1087.50 & 800 \\
\hline & & 1.2 & 1293.50 & 830 \\
\hline & & 1.5 & 1662.50 & 875 \\
\hline & \multirow{4}{*}{3.0} & 0.5 & 662.50 & 725 \\
\hline & & 1.0 & 1087.50 & 800 \\
\hline & & 1.2 & 1408.70 & 830 \\
\hline & & 1.5 & 2112.50 & 875 \\
\hline
\end{tabular}

Table 4.4. Value of $E\left[C_{w}(x, \alpha, n)\right]$ for different combinations of $\delta$ and $x$ for $\alpha=0, \alpha=1$ when $\gamma<1$

\begin{tabular}{|c|c|c|c|c|}
\hline$\gamma$ & $\delta$ & $x$ & $\alpha=0$ & $\alpha=1$ \\
\hline \multirow{20}{*}{$\gamma<1$} & \multirow{4}{*}{0.4} & 0.3 & 829.62 & 695 \\
\hline & & 0.5 & 915.64 & 725 \\
\hline & & 1.0 & 1087.50 & 800 \\
\hline & & 1.5 & 1232.94 & 875 \\
\hline & \multirow{4}{*}{0.7} & 0.3 & 754.71 & 695 \\
\hline & & 0.5 & 858.73 & 725 \\
\hline & & 1.0 & 1087.50 & 800 \\
\hline & & 1.5 & 1293.78 & 875 \\
\hline & \multirow{4}{*}{1.0} & 0.3 & 702.50 & 695 \\
\hline & & 0.5 & 812.50 & 725 \\
\hline & & 1.0 & 1087.50 & 800 \\
\hline & & 1.5 & 1362.50 & 875 \\
\hline & \multirow{4}{*}{2.0} & 0.3 & 618.50 & 695 \\
\hline & & 0.5 & 712.50 & 725 \\
\hline & & 1.0 & 1087.50 & 800 \\
\hline & & 1.5 & 1662.50 & 875 \\
\hline & \multirow{4}{*}{3.0} & 0.3 & 593.30 & 695 \\
\hline & & 0.5 & 662.50 & 725 \\
\hline & & 1.0 & 1087.50 & 800 \\
\hline & & 1.5 & 2112.50 & 875 \\
\hline
\end{tabular}


감소시키는 방법이었다. 이러한 주기적 예방보전정책은 새로운 시스템에 관하여는 현재까지 많은 연구 가 진행되어 왔으나 중고 시스템에 관한 연구는 극히 제한적이었으며 이러한 관점에서 이 논문은 매우 중요한 의미를 가진다고 하겠다. 현대사회에서 중고 시스템 시장은 지속적인 성장과 높은 경쟁성을 보 이고 있으며 따라서 소비자뿐 아니라 판매자 또한 다양한 측면에서 높은 신뢰도를 가지는 중고 시스템을 제공하고 구매하기 위한 정책에 관심을 가지고 있으며 이러한 경향은 앞으로도 증가될 것으로 예상된다. 이 논문에서는 보증기간동안 발생되는 모든 비용을 판매자가 부담하는 보증정책을 고려하였는데 따라서 판매자의 관점에서 보증비용을 줄이기 위한 최적 보전정책의 필요성이 제기된다. 주기적 예방보전 시에 는 시스템의 고장률을 향상지수 $\alpha$ 만큼 감소시켜 시스템의 신뢰도를 향상시키는 정책을 가정하였고 비용 모형을 정의함으로서 보증기간 동안에 소요되는 판매자의 전체 보증비용에 대한 기댓값을 계산하는 수 학적 공식을 유도하여 이러한 비용을 최소화하는 최적의 $\alpha$ 값을 계산 하였다. 또한 실직적인 수치적 예 를 위해 와이블 분포를 사용하여 주요 모수인 $\gamma, \delta, x$ 의 값의 변화가 전체 보증비용에 미치는 영향을 조 사하기 위하여 민감도분석을 하였다.

이번 논문에서 고려한 보증정책과 주기적 예방보전정책 이외에도 중고 시스템에 적용 가능한 다양한 형 태의 정책들이 가능할 것으로 생각되며 앞으로 추가적인 연구가 필요하다고 본다. 또한 판매자의 관점 에서가 아닌 소비자 측면에서의 보증기간 종료 이후의 주기적 예방보전에 대한 연구도 매우 흥미로운 과 제가 될 것으로 생각되며 수리비용에 대해서도 본 논문에서 고려한 형태 이외의 다른 비용모형을 고려할 수 있으며 주기적 보전 시점에서의 고장률 감소에 대하여도 또 다른 모형을 가정한다면 보다 일반적인 연구결과를 얻을 수 있을 것으로 기대된다.

\section{References}

Blischke, W. R. and Murthy, D. N. P. (1994). Warrant Cost Analysis0, Marcel Dekker, New York.

Blischke, W. R. and Murthy, D. N. P. (1996). Product Warranty Handbook, Marcel Dekker, New York.

Blischke, W. R. and Murthy, D. N. P. (2000). Strategic warranty management: A life-cycle approach, IEEE Transactions on Engineering Management, 47, 40-54.

Chien, Y. H. (2008). A general age replacement model with minimal repair under renewing free replacement warranty, European Journal of Operational Research, 186, 1046-1058.

Jung, K. M. (2009). Replacement model for repairable system after repair warranty, Korean Data Analysis Society, 11, 3355-3365.

Jung, K. M., Han, S. S. and Park, D. H. (2008). Optimization of cost and downtime for replacement model following the expiration of warranty, Reliability Engineering \& System Safety, 93, 995-1003.

Jung, K. M. and Park, D. H. (2003). Optimal maintenance policies during the post-warranty period, Reliability Engineering \& System Safety, 82, 173-185.

Murthy, D. N. P. and Jack, N. (2003). Warranty and maintenance, Handbook of Reliability Engineering, $525-534$.

Nakagawa, T. (1986). Periodic and sequential preventive maintenance policies, Journal of Applied Probability, 23, 536-542.

Sahin, I. and Polatoglu, H. (1996). Maintenance strategies following the expiration of warranty, IIE Transactions, 45, 220-228.

Zhang, F. and Jardine, A. K. S. (1998). Optimal maintenance models with minimal repair, periodic overhaul and complete, IIE Transactions, 30, 109-1119. 


\title{
주기적인 유지보수 계획에 따른 중고제품에 대한 최적 향상수준
}

\author{
김대경 $a \cdot$ 김진우 $^{b} \cdot$ 박동호 $^{c, 1}$ \\ ${ }^{a}$ 전북대학교 통계학과, ${ }^{b}$ 한림대학교 통계학과, ${ }^{c}$ 한림대학교 연구교수
}

(2012년 12월 10일 접수, 2013년 1월 11일 수정, 2013년 1월 23일 채택)

\section{요 약}

중고제품 특히 고가인 중고품에 대한 점증하는 수요로 인하여 그러한 제품에 대한 보증과 보전정책이 최근 제품의 신 뢰성을 향상시키기 위해 연구되고 있다. 이 논문에서는 사용한 년 수 $x$ 에서 고객에 의해서 구입된 중고제품에 대한 주기적인 유지보수모형을 연구하였다. 구입할 때 판매자는 제품의 고장율을 줄이기 위해서 그리고 각각의 유지보수 가 수행되고 난 이후에 신뢰성을 향상시키기 위해서 정해진 보증기간을 제공한다. 만일 연속적인 유지보수 사이에서 고장이 일어난다면 단지 최소수리가 행해진다. 보증정책에 대해서 보증기간동안에 주기적인 유지보수 점검과 더불 어 각 고장에 관해서는 무상 비재생수리를 한다. 따라서 이러한 보증정책 하에서 보증기간에 일어난 모든 유지보수 와 수리비용은 판매자에게 부과된다. 제안된 주기적인 유비보수 계획에 대해서 보증기간 동안에 판매자에게 부과된 기대 총비용을 계산하기 위한 모형과 판매자의 측면에서 총기대보증비용을 최소화하기 위한 각 유지보수에서 고장율 의 최적향상수준을 유도한다. 또한 제안된 방법들에 근거해서 최적향상수준에 대한 수치적인 결과를 제시한다.

주요용어: 중고제품, 보증기간, 최소수리, 비재생수리, 최적향상수준, 총기대비용.

이 논문은 2011년도 정부(교육과학기술부)의 재원으로 한국연구재단의 지원을 받아 수행된 기초연구사업임 (2011-0022398).

${ }^{1}$ 교신저자: (200-702) 강원도 춘천시 한림대학길 1 , 산학협력단, 한림대학교, 연구교수.

E-mail: dhpark@hallym.ac.kr 